\title{
Crowding y overtourism en la literatura de turismo y cruceros
}

\begin{abstract}
Fernando J. Garrigos-Simon ${ }^{a}$, Yeamduan Narangajavana Kaosiri ${ }^{b}$, Silvia Sanz-Blas ${ }^{c}$, Sofia Estelles-Miguel $^{a}$, Juan Vicente Oltra-Gutierrez' ${ }^{a}$, Daniela Buzova ${ }^{\text {c }}$

${ }^{a}$ Universitat Politècnica de Valencia, Valencia, España fgarrigos@doe.upv.e, ${ }^{b}$ Universitat Jaume I, Castellón, España ynaranga@uji.es, 'Universidad de Valencia, Valencia, España silvia.sanz@uv.es, daniela.buzova@uv.es
\end{abstract}

\begin{abstract}
Resumen
El objeto de este artículo es realizar un pequeño análisis de la situación de la literatura de Crowding y Overtourism en el sector turístico. Además, el trabajo hace un repaso especial a esta literatura enfatizando en la literatura de cruceros. Utilizando como base la Web of Science, el estudio realiza un breve análisis bibliomético de esta literatura. El propósito es conocer el estado del arte y principales tendencias en esta literatura. Además el trabajo pretende sentar las bases para un posterior análisis en profundidad de esta literatura. El trabajo tiene repercusiones para investigadores, dado que analiza las carencias en la literatura y las nuevas tendencias futuras. Además, el trabajo puede ayudar a profesionales para observar nuevas prácticas y cuestiones que deben desarrollar e implementar en empresas y organizaciones.
\end{abstract}

Palabras clave: Crowding, Overtourism, Turismo, Cruceros, Bibliométrico.

\section{Abstract}

The purpose of this article is to make a small analysis of the situation of Crowding and Overtourism literature in the tourism sector. In addition, the paper makes a special review of this literature with emphasis on cruise ship literature. Using the Web of Science as a basis, the study makes a brief bibliometric analysis of this literature. The purpose is to know the state of the art and main trends in this literature. In addition, the work aims to lay the foundations for a subsequent in-depth analysis of this literature. The work has implications for researchers, as it analyses gaps in the literature and new future trends. In addition, the work can help professionals to observe new practices and issues to be developed and implemented in companies and organizations.

Keywords: Crowding, Overtourism, Tourism, Cruises, Bibliometric. 


\section{Introducción}

El turismo de crucero es de crucial importancia para determinados destinos turísticos, especialmente para diversas islas especializadas en este tipo de desarrollos como son múltiples islas del Caribe o del pacífico. A su vez, su relevancia está siendo esencial para destinos turísticos clásicos reconvertidos en puertos de embarque, y que a partir de los cruceros han creado verdaderos hubs logísticos. Es el caso de Miami en Estados Unidos, Barcelona y Venecia en el Mediterráneo, o Copenhague en Dinamarca.

Sin embargo como toda industria, la industria de cruceros tiene efectos deseables e indeseables, apuntados ya por McKee y Mamoozadeh (1994) o Hall y Braithwaite (1990) a finales del siglo pasado. Entre ellos se encuentran efectos económicos, sociales e incluso culturales en los destinos y sus comunidades, o impactos medioambientales en los ecosistemas marinos o costeros. En este sentido, diversa literatura observa el efecto de los cruceristas en la sostenibilidad socio-cultural o medioambiental de los destinos (Cerveny et al., 2020), mientras por ejemplo atendiendo a aspectos económicos, la literatura menciona el posible efecto "crowding-out" en la demanda "stay-over", es decir, el efecto expulsión o sustitución del alojamiento o estancias (aunque los estudios no son concluyentes, al depender de los destinos concretos) (Bresson y Logossah, 2011).

Más en el campo del marketing, la literatura ha incidido en la relevancia del "crowding" o multitud en las percepciones de turistas y otros agentes. En este sentido, la literatura turística ha observado el "crowding" tanto como un aspecto positivo, ligado a la popularidad o la fama (Petr, 2009), como, por el contrario, una fuente de reacciones negativas. Las reacciones negativas están asociadas esencialmente a la capacidad de carga de los destinos (Garrigos-Simon et al, 2004), fundamentalmente a la capacidad de carga social (Jurado et al., 2013), o a determinados efectos socio-psicológicos ligados al "crowding" u "overtourism" (Gössling, et al., 2020) o "tourismphobia" (Veríssimo et al., 2020). Ello podría conducir a experiencias desagradables que disminuyen la satisfacción del turista o la sobresaturación de destinos (Simancas-Cruz y Peñarrubia Zaragoza, 2019). También podría estar ligado al hecho de no preferir o querer evitar ciertos turistas aquellos destinos u organizaciones multitudinarias, al causarles insatisfacción o actitudes negativas (Dowling, 2006; Luque-Gil et al, 2018).

En el área de cruceros la literatura también ha incidido en estos aspectos, por su efecto tanto en destinos como en la imagen de los cruceros, o el desempeño de estos. En este sentido, la literatura postula sobre el efecto negativo que puede tener la percepción de multitud o sobrecapacidad causada o no por los cruceristas en los residentes u otros turistas (Marušić et al., 2008), en el valor percibido de la marca de lujo en cruceros (Hyun. y Kim, 2015), la percepción de atractividad de los destinos por parte de los turistas, incluyendo la distinta 
tolerancia hacia ella por parte de los cruceristas (Jacobsen et al., 2019), o la percepción de excursiones llenas de gente en la satisfacción de los cruceristas (Buzova et al., 2019), hechos que obviamente requerirían de mejoras en la gestión de flujos de visitantes por parte de organizaciones y destinos.

No obstante, aunque ciertos estudios pioneros han intentado realizar recientemente un análisis sobre esta situación en el campo turístico (Gössling, et al., 2020; Veríssimo et al., 2020), los estudios sobre el tema son incipientes, y más en el área de cruceros, donde los estudios teóricos y prácticos son muy reducidos y limitados. El propósito de este trabajo es realizar un pequeño estudio de la principal literatura en esta área, su estructura y evolución.

\section{Material y Métodos}

Este Trabajo utiliza el análisis bibliométrico. Este método analiza cuantitativamente el contenido bibliográfico. El método es reconocido por su habilidad para explorar la estructura de la investigación en general, y en concreto la investigación en turismo. Esta metodología además es ampliamente utilizada para identificar el desarrollo de un campo y sus tendencias. A su vez, el análisis bilbiométrico permite extraer y vislumbrar conclusiones muy valiosas, utilizando para ello información objetiva de una manera muy amigable (Garrigos-Simon et al., 2018).

El proceso de investigación se basa en datos extraídos de la WoS Core Collection. El trabajo utiliza esta base de datos por ser la base de datos más selectiva e influyente en la investigación académica.

De esta base de datos recopilamos, en primer lugar, todos los documentos que utilizaron las palabras clave "crowding" u "overtourism" en el área de "Hospitality, leisure, sport \& tourism", enfocándonos posteriormente en todos los documentos de la base de datos que utilizaron las palabras clave "crowding" y "overtourism" en conjunción con "cruise", con objeto de seleccionar la literatura especifica en cruceros. Estas prácticas son muy comunes en la literatura bilibométrica (Garrigos-Simon et al., 2018). La población considera todos los años hasta el 31 de diciembre de 2019. El proceso de extracción de los datos tuvo lugar en febrero de 2020. Posteriormente, los datos se filtraron para considerar solamente artículos, revisiones, cartas y notas, con objeto de poder concentrarnos en los principales documentos. Las muestras finales consideran 394 documentos para la literatura general, y 30 documentos para la literatura sobre cruceros. 
El trabajo utiliza sólo alguno de los indicadores más comunes en la literatura bibliométrica, como el número total de artículos y citas para medir la productividad e impacto del área. A su vez, analiza los diez trabajos más citados en el área general.

\section{Resultados}

El trabajo exploratorio realiza dos tipos de análisis. En primer lugar, el trabajo presenta el estado actual y progreso de ambos grupos de literatura. En segundo lugar, el trabajo revisa los artículos más citados en el campo.

\subsection{Estado, Evolución y Estructura de la literatura de Crowding y Overtourism, tanto en la literatura general turística como en la relacionada con cruceros}

El primer trabajo sobre "crowding" u "overtourism" publicado en la WoS en el área de "Hospitality, leisure, sport \& tourism" fue el trabajo de Hillery y Lincoln, publicado en 1978. No fue hasta 20 años después, cuando este tópico recibió su primer análisis sobre cruceros, mencionado en la WoS, concretamente con el trabajo de Malik (1998), aunque con una perspectiva ajena a nuestro trabajo, mientras que el primer artículo sobre cruceros en el área de "Hospitality, leisure, sport \& tourism" fue el de Bresson y Logossah (2011), ya en nuestra perspectiva de trabajo sobre cruceros (debemos mencionar que hasta la fecha, en el área de turismo sobre cruceros y este tópico, sólo hay publicados 6 trabajos, dos de ellos en 2019).

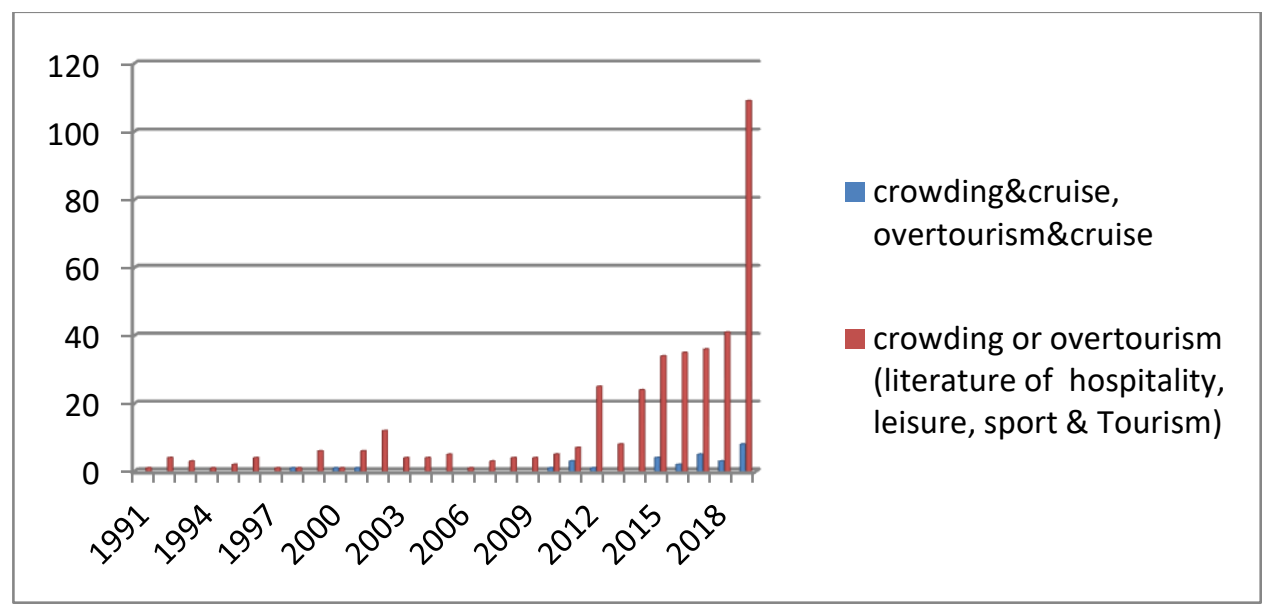

Fig.1. Publicaciones anuales en la WoS de la literatura sobre Crowding y Overtourism. En Rojo las publicaciones en la literatura de "hospitality, leisure, sport \& tourism", y en azul en la literatura relacionada con cruceros. Fuente WoS. 
No obstante, el número de documentos (artículos, revisiones, cartas y notas) experimentó un crecimiento exponencial, sobre todo en la última década. Así, en la literatura turística a partir de 2012 los trabajos publicados superan la veintena anualmente (excepto en 2013), llegando a más de 100 trabajos en 2019. A su vez, en la literatura de cruceros en general, aunque el desarrollo es posterior y todavía incipiente, los trabajos ya son constantes anualmente en el último lustro, con 8 trabajos ya en 2019 (Fig. 1).

El análisis de la estructura de citas de los documentos en nuestra área muestra el impacto de esta nueva área. Las tablas 1 y 2 observan la estructura de citas en los dos grupos de literatura. Observamos, a este respecto, que en ambos grupos de literatura más de un tercio de los artículos reciben 10 o más citas, con un artículo con más de 300 citas en la literatura de turismo en general, mientras que el trabajo más citado en la literatura de cruceros sobrepasa el centenar de citas.

Tabla 1. Estructura general de citas de artículos sobre "crowding" y "overtourism" en la literatura de "Hospitality, leisure, sport \& tourism"

\begin{tabular}{|c|c|c|c|c|}
\hline $\begin{array}{l}\text { Número de } \\
\text { citas }\end{array}$ & $\begin{array}{l}\text { Número de } \\
\text { artículos }\end{array}$ & $\begin{array}{l}\text { Número } \\
\text { acumulado de } \\
\text { artículos }\end{array}$ & $\%$ Articlulos & $\begin{array}{c}\% \text { Acumulado } \\
\text { de artículos }\end{array}$ \\
\hline$\geq 250$ & 1 & 1 & 0,25 & 0,25 \\
\hline$\geq 100$ & 5 & 6 & 1,27 & 1,52 \\
\hline$\geq 50$ & 18 & 24 & 4,57 & 6,09 \\
\hline$\geq 25$ & 41 & 65 & 10,41 & 16,50 \\
\hline$\geq 10$ & 71 & 136 & 18,02 & 34,52 \\
\hline$<10$ & 258 & 394 & 65,48 & 100,00 \\
\hline Total & 394 & & & \\
\hline
\end{tabular}

Fuente: Elaboracion propia basada en WoS 2020

Tabla 2. Estructura general de citas de articulos sobre crowding y overtourism en la literatura de cruceros

\begin{tabular}{|l|r|r|r|r|}
\hline \multicolumn{1}{|c|}{$\begin{array}{c}\text { Crowding \& Overtourism in tourism literature } \\
\text { citas }\end{array}$} & $\begin{array}{c}\text { Número de } \\
\text { artículos }\end{array}$ & $\begin{array}{c}\text { Número } \\
\text { acumulado de } \\
\text { artículos }\end{array}$ & \% Articlulos & $\begin{array}{c}\text { \% Acumulado } \\
\text { de artículos }\end{array}$ \\
\hline$\geq 50$ & 1 & $\mathbf{1}$ & $\mathbf{3 , 3 3}$ & $\mathbf{3 , 3 3}$ \\
\hline$\geq 25$ & $\mathbf{2}$ & $\mathbf{3}$ & $\mathbf{6 , 6 7}$ & $\mathbf{1 0 , 0 0}$ \\
\hline$\geq 10$ & $\mathbf{8}$ & $\mathbf{1 1}$ & $\mathbf{2 6 , 6 7}$ & $\mathbf{3 6 , 6 7}$ \\
\hline$<10$ & $\mathbf{1 9}$ & $\mathbf{3 0}$ & $\mathbf{6 3 , 3 3}$ & $\mathbf{1 0 0 , 0 0}$ \\
\hline Total & $\mathbf{3 0}$ & \multicolumn{3}{|c|}{} \\
\hline
\end{tabular}

Fuente: Elaboracion propia basada en WoS 2020 


\subsection{Artículos más citados sobre Crowding y Overtourism en la literatura turística}

En la tabla 3, observamos los artículos sobre “crowding” y "overtourism” más citados en la literatura turística. La mayoría de estos artículos inciden en normativas sobre crowding y el impacto del "crowding" y el "overtourism" en las percepciones de grupos de interés relacionados con actividades deportivas. En este aspecto, vemos una combinación de artículos tanto en algunas de las revistas líderes de la literatura turística, como Annals of Tourism Research y Tourism Management, como en otras que inciden directa o indirectamente en la literatura deportiva.

Tabla 3. Principales artículos sobre "crowding" y "overtourism" en la literature de "Hospitality, leisure, sport \& tourism"

\begin{tabular}{|c|c|c|c|c|c|c|}
\hline $\mathbf{R}$ & Revista & $\begin{array}{l}\text { Citas } \\
\text { tota- } \\
\text { les }\end{array}$ & Artículo & Autores & \begin{tabular}{|c|} 
Año de \\
Publica- \\
ción
\end{tabular} & $\begin{array}{c}\text { Citas por } \\
\text { año } \\
\end{array}$ \\
\hline 1 & $\begin{array}{l}\text { Annals of } \\
\text { Tourism } \\
\text { Research }\end{array}$ & 309 & $\begin{array}{l}\text { Residents' attitudes toward tourism } \\
\text { development }\end{array}$ & $\begin{array}{l}\text { Teye, V; Sonmez, } \\
\text { SF; Sirakaya, E }\end{array}$ & 2002 & 18,18 \\
\hline 2 & $\begin{array}{l}\text { Psychology of } \\
\text { Sport and } \\
\text { Exercise }\end{array}$ & 222 & $\begin{array}{l}\text { The influence of crowd noise and } \\
\text { experience upon refereeing decisions in } \\
\text { football }\end{array}$ & $\begin{array}{l}\text { Nevill, } \\
\text { AM; Balmer, } \\
\text { NJ; Williams, AM }\end{array}$ & 2002 & 13,06 \\
\hline 3 & $\begin{array}{l}\text { Sport } \\
\text { Psychologist }\end{array}$ & 182 & $\begin{array}{l}\text { Factors affecting Olympic performance: } \\
\text { Perceptions of athletes and coaches } \\
\text { from more and less successful teams }\end{array}$ & $\begin{array}{l}\text { Gould, D; Guinan, } \\
\text { D; Greenleaf, C; et } \\
\text { ál.. }\end{array}$ & 1999 & 9,10 \\
\hline 4 & $\begin{array}{l}\text { Journal of } \\
\text { Leisure } \\
\text { Research }\end{array}$ & 115 & $\begin{array}{l}\text { Effect of activity involvement and place } \\
\text { attachment on recreationists' } \\
\text { perceptions of setting density }\end{array}$ & $\begin{array}{l}\text { Kyle, G; Graefe, } \\
\text { A; Manning, R; et } \\
\text { ál.. }\end{array}$ & 2004 & 7,67 \\
\hline 5 & $\begin{array}{l}\text { Leisure } \\
\text { Sciences }\end{array}$ & 104 & $\begin{array}{l}\text { Crowding norms at frontcountry sites: A } \\
\text { visual approach to setting standards of } \\
\text { quality }\end{array}$ & $\begin{array}{l}\text { Manning, } \\
\text { RE; Lime, } \\
\text { DW; Freimund, } \\
\text { WA; et ál.. }\end{array}$ & 1996 & 4,52 \\
\hline 6 & $\begin{array}{l}\text { Sport } \\
\text { Psychologist }\end{array}$ & 101 & $\begin{array}{l}\text { A survey of US Olympic coaches: } \\
\text { Variables perceived to have influenced } \\
\text { athlete performances and coach } \\
\text { effectiveness }\end{array}$ & $\begin{array}{l}\text { Gould, D; Guinan, } \\
\text { D; Greenleaf, C; et } \\
\text { ál.. }\end{array}$ & 2002 & 5,94 \\
\hline 7 & $\begin{array}{l}\text { Tourism } \\
\text { Management }\end{array}$ & 97 & $\begin{array}{l}\text { A qualitative and quantitative } \\
\text { assessment of Hong Kong's image as a } \\
\text { tourist destination }\end{array}$ & $\begin{array}{l}\text { Choi, WM; Chan, } \\
\text { A; Wu, J }\end{array}$ & 1999 & 4,85 \\
\hline 8 & $\begin{array}{l}\text { Leisure } \\
\text { Sciences }\end{array}$ & 88 & $\begin{array}{l}\text { Crowding norms: Alternative } \\
\text { measurement approaches }\end{array}$ & $\begin{array}{l}\text { Manning, } \\
\text { RE; Valliere, } \\
\text { WA; Wang, B }\end{array}$ & 1999 & 4,40 \\
\hline 9 & $\begin{array}{l}\text { Tourism } \\
\text { Management }\end{array}$ & 87 & $\begin{array}{l}\text { But are tourists satisfied? Importance- } \\
\text { performance analysis of the whale shark } \\
\text { tourism industry on Isla Holbox, Mexico }\end{array}$ & $\begin{array}{l}\text { Ziegler, } \\
\text { Jackie; Dearden, } \\
\text { Philip; Rollins, } \\
\text { Rick }\end{array}$ & 2012 & 12,43 \\
\hline 10 & $\begin{array}{l}\text { Journal of } \\
\text { Leisure } \\
\text { Research }\end{array}$ & 85 & $\begin{array}{l}\text { Differential-effects of past experience, } \\
\text { commitment, and life-style dimensions } \\
\text { on river use specialization }\end{array}$ & $\begin{array}{l}\text { Kuentzel, } \\
\text { wf; mcdonald, cd }\end{array}$ & 1992 & 3,15 \\
\hline
\end{tabular}

Fuente: Elaboracion propia basada en WoS 2020 


\section{Conclusiones}

Este trabajo exploratorio ha incidido en la importancia del "crowding" y el "overtourism" en la literatura turística, analizando su traslación a la literatura centrada en los cruceros.

El trabajo ha observado que aunque la literatura es todavía pionera, los estudios han experimentado un desarrollo muy importante recientemente, considerándose el área como un área de "moda". El estudio ha detectado diversas carencias, y la todavía inexistencia de la translación del tema de estudio al análisis del desarrollo de cruceros.

Este trabajo es limitado, dado que la propia extensión del mismo ha impedido el uso de instrumentos bibliográficos más desarrollados. En este sentido, posteriores trabajos podrían ampliar este trabajo con el uso de técnicas bibliométricas más sofisticadas, como el análisis de fuentes o instrumentos como el análisis de citas, coocurrencias u otros.

No obstante, dada la incipiencia, pero también explosivo crecimiento del área, el trabajo es importante para autores y profesionales, dado que para los primeros plantea múltiples áreas de desarrollo, mientras que para los segundos plantea cuestiones en las que deberían incidir en la gestión y el marketing de organizaciones turísticas y cruceros.

\section{Agradecimientos}

Los autores agradecen financiación por parte de la Universitat Politècnica de Valencia, la Universitat Jaume I y la Universitat de València.

\section{Referencias}

Bresson, G., \& Logossah, K. (2011). Crowding-out effects of cruise tourism on stay-over tourism in the Caribbean: Non-parametric panel data evidence. Tourism Economics, 17(1), 127-158.

Buzova, D., Sanz-Blas, S., \& Cervera-Taulet, A. (2019). „Tour me onshore ${ }^{e e}$ understanding cruise tourists evaluation of shore excursions through text mining. Journal of Tourism and Cultural Change, 17(3), 356-373.

Cerveny, L. K., Miller, A., \& Gende, S. (2020). Sustainable cruise tourism in marine world heritage sites. Sustainability, 12(2), 611.

Dowling, R. K. (Ed.). (2006). Cruise ship tourism. Cabi.

Garrigos-Simon, F.J.,, Narangajavana-Kaositi, Y., \& Palacios-Marques, D. (2004). Carrying capacity in the tourism industry: a case study of Hengistbury Head. Tourism management, 25(2), 275-283.

Garrigos-Simon, F. J., Narangajavana-Kaosiri, Y., \& Lengua-Lengua, I. (2018). Tourism and sustainability: A bibliometric and visualization analysis. Sustainability, 10(6), 1976. 
Gössling, S., McCabe, S., \& Chen, N. C. (2020). A socio-psychological conceptualisation of overtourism. Annals of Tourism Research, 84, 102976.

Hall, J. A., \& Braithwaite, R. (1990). Caribbean cruise tourism: a business of transnational partnerships. Tourism Management, 11(4), 339-347.

Hillery, G. A., \& Lincoln, A. J. (1978). Leisure, Freedom, and Crowd Behavior. Journal of Leisure Research, 10(3), 219-225.

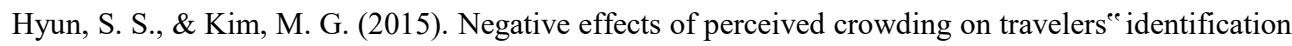
with cruise brand. Journal of Travel \& Tourism Marketing, 32(3), 241-259.

Jacobsen, J. K. S., Iversen, N. M., \& Hem, L. E. (2019). Hotspot crowding and over-tourism: Antecedents of destination attractiveness. Annals of Tourism Research, 76, 53-66.

Jurado, E. N., Damian, I. M., \& Fernández-Morales, A. (2013). Carrying capacity model applied in coastal destinations. Annals of Tourism Research, 43, 1-19.

Luque-Gil, A. M., Gómez-Moreno, M. L., \& Peláez-Fernández, M. A. (2018). Starting to enjoy nature in Mediterranean mountains: Crowding perception and satisfaction. Tourism management perspectives, 25, 93-103.

Marušić, Z., Horak, S., \& Tomljenović, R. (2008). The socioeconomic impacts of cruise tourism: A case study of Croatian destinations. Tourism in Marine Environments, 5(2-3), 131-144.

McKee, D. L. and A. Mamoozadeh, 1994, Cruise ships in the third world: developmental versus corporate financial objectives, in McKee, D.L, External Linkages and Growth in Small Economies, Greenwood, Westport, Conn. and London, 91-101

Malik, O. (1998). Battle for the Public Mind. The World Today, 54(10), 263-265.

Petr, C. (2009). Fame is not always a positive asset for heritage equity! Some clues from buying intentions of national tourists. Journal of Travel \& Tourism Marketing, 26(1), 1-18.

Simancas Cruz, M., \& Peñarrubia Zaragoza, M. P. (2019). Analysis of the accommodation density in coastal tourism areas of insular destinations from the perspective of overtourism. Sustainability, 11(11), 3031.

Veríssimo, M., Moraes, M., Breda, Z., Guizi, A., \& Costa, C. (2020). Overtourism and tourismphobia: A systematic literature review. Tourism: An International Interdisciplinary Journal, 68(2), 156-169. 